\title{
Representation of Quantum Field Theory by Elementary Quantum Information
}

\author{
Martin Kober* \\ Frankfurt Institute for Advanced Studies (FIAS), Johann Wolfgang Goethe-Universität, \\ Ruth-Moufang-Strasse 1, 60438 Frankfurt am Main, Germany
}

(Dated: October 25, 2018)

\begin{abstract}
In this paper is considered relativistic quantum field theory expressed by elementary units of quantum information as they are considered as fundamental entity of nature by Carl Friedrich von Weizsaecker. Through quantization of a Weyl spinor describing an elementary unit of quantum information and consisting of four real components one obtains four pairs of creation and annihilation operators acting in a tensor space of states containing many units of quantum information. There can be constructed position and momentum operators from the creation and annihilation operators and based on these operators the Poincare group can be represented in this abstract tensor space of quantum information. A general state in the tensor space can be mapped to a state in Minkowski space-time by using the position representation of the eigenstates of the occupation number operators which correspond to the eigenstates of the harmonic oscillator. This yields a description of relativistic quantum mechanics. Quantization of the coefficients of a general state in the tensor space leads to many particle theory and thus to quantum field theory.
\end{abstract}

\section{INTRODUCTION}

In his program of a reconstruction of physics Carl Friedrich von Weizsaecker makes the attempt to reconstruct physics from general quantum theory, which is considered as general theory of human knowledge about nature and accordingly quantum theory is interpreted as consequence of general postulates about the structure of human knowledge [1],[2], [3],[4],[5], [6], [7], [8]. This means nothing else but that within this program physical objects and their properties shall be inferred from abstract quantum information interpreted as fundamental entity of nature, which can be resolved into binary alternatives called ur-alternatives by von Weizsaecker. This denotation arises from the fundamental role the binary alternatives play in this approach, where is introduced a tensor space of many ur-alternatives, which are elementary units of quantum information. According to von Weizsaecker from the mathematical structure of the uralternatives, which can be described by Weyl spinors, the existence of position space as representation of physical relations between objects consisting of this elementary quantum information can be derived. This property is related to the fact that the symmetry group of two dimensional complex Hilbert spaces is the special unitary group in two dimensions, the $S U(2)$, which is isomorphic to the $S O(3)$, the rotation group in a three dimensional real vector space as it is realized in nature. The group of general linear transformations in two complex dimensions, the $S L(2, \mathbb{C})$, is isomorphic to the Lorentz group, which means that the structure of Minkowski space-time is also implicitly contained in a two dimensional complex vector space [9], 10] describing the possible states of an elementary unit of quantum information.

In the present paper is considered quantum field theory

*Electronic address: kober@fias.uni-frankfurt.de represented by elementary units of quantum information as they are considered as fundamental entity of nature by von Weizsaecker. Concerning the basic concepts it is treated according to von Weizsaecker. There is considered a tensor space describing many units of quantum information, but it is developed a new method to obtain a representation of the states of the tensor space in usual space-time. Since every physical state contains information and information can be represented by binary alternatives, it has to be possible to describe any quantum state including states of any particle or quantum field by elementary units of quantum information. If one wants to describe a physical object, a particle for example, one needs of course more than just single units of quantum information. Therefore it is necessary to consider a Hilbert space of quantum states containing many quantum information. Such a space is obtained by quantizing the Weyl spinor describing a single unit of quantum information. By quantizing the Weyl spinor the several components of the Weyl spinor describing the state of the unit of quantum information become creation and annihilation operators. These creation and annihilation operators create and annihilate units of quantum information in the corresponding basis state of a single unit of quantum information the component refers to and therefore one is led to a Hilbert space, which is the tensor space of many units of quantum information and thus represents the tensor space referring to the vector space consisting of the possible states of single Weyl spinors. The creation and annihilation operators act in this Hilbert space and transform the basis states, which are the states with sharp occupation numbers of units of quantum information in the several possible basis states of a single unit of quantum information, into each other. The state of a single particle can be represented by a general state in such a Hilbert space of many units of quantum information.

If quantum field theory shall be derived or at least be represented by quantum information, it is of course necessary to relate states in the tensor space of elementary 
quantum information to states referring to space-time. More exactly the states in the tensor space have to be represented in space-time. The method to achieve a transition from tensor space to space-time as it is considered in this paper consists in the definition of four pairs of position and momentum operators from the four pairs of creation and annihilation operators obtained from the quantization of single units of quantum information. As already mentioned a basis of states in the tensor space of elementary units of quantum information is given by all occupation numbers of the several basis states of an elementary unit of quantum information. These states are eigenstates of the occupation number operators and thus correspond to the eigenstates of the more dimensional harmonic oscillator in quantum mechanics, which can be represented in position space. Accordingly a general state in the tensor space of elementary quantum information, which can be expressed by a superposition of eigenstates of the occupation number operators, can be mapped to a state in space-time by representing the eigenstates of the harmonic oscillator in space-time. Thus the states in the tensor space obtained by the quantization of the elementary units of quantum information can indeed be represented as wave functions in space-time. With the position and momentum operators defined by the creation and annihilation operators in the tensor space of elementary quantum information can be constructed the generators of the Poincare group, which can thus be represented in the tensor space. Since particles are described by irreducible representations of the Poincare group in quantum field theory [11],[12], the states in tensor space appearing as wave functions in space-time can be considered as states of particles. According to usual field quantization the corresponding quantum field theory referring to elementary quantum information is obtained by quantizing the coefficients of a general state of a single particle, which corresponds to the wave function in usual quantum mechanics. This leads to a theory of many particles and thus to quantum field theory. The basis states of the new Hilbert space are defined by the occupation numbers referring to the basis states in the tensor space of elementary quantum information.

\section{TENSOR SPACE OF ELEMENTARY QUANTUM INFORMATION}

A single unit of quantum information is described by a Weyl spinor and thus by a normalized two dimensional complex vector,

$$
u=\left(\begin{array}{l}
u_{1} \\
u_{2}
\end{array}\right)=\left(\begin{array}{l}
a+b i \\
c+d i
\end{array}\right)
$$

which contains four real components denoted by $a, b, c$ and $d$, for which holds of course the following relation as normalization condition: $a^{2}+b^{2}+c^{2}+d^{2}=1$. Real physical objects naturally contain much quantum information and because of this there has to be considered a Hilbert space of states containing much quantum information. To obtain such a Hilbert space of states containing much quantum information and not just a single unit, it is necessary to quantize the elementary unit of quantum information again, which means that it becomes an operator, $u \rightarrow \hat{u}$. This quantization is performed as usual by postulating canonical commutation relations for the unit of quantum information. Especially there is postulated the following commutation relation:

$$
\left[\hat{u}_{r}, \hat{u}_{s}^{\dagger}\right]=\delta_{r s} \quad, \quad r, s=1,2,
$$

defining the quantum properties of the operator of the elementary unit of quantum information $\hat{u}$ and its hermitian adjoint $\hat{u}^{\dagger}$. This commutation relation (2) can be realized, if the real components of the corresponding Weyl spinor defined above, $a, b, c$ and $d$, become operators fulfilling the following commutation relations:

$$
\left[\hat{a}, \hat{a}^{\dagger}\right]=1, \quad\left[\hat{b}, \hat{b}^{\dagger}\right]=1, \quad\left[\hat{c}, \hat{c}^{\dagger}\right]=1, \quad\left[\hat{d}, \hat{d}^{\dagger}\right]=1 .
$$

All other commutators between the operators $\hat{a}, \hat{b}, \hat{c}$ and $\hat{d}$ as well as the corresponding hermitian adjoint operators $\hat{a}^{\dagger}, \hat{b}^{\dagger}, \hat{c}^{\dagger}$ and $\hat{d}^{\dagger}$ are assumed to vanish. These operators accordingly behave as creation and annihilation operators creating and annihilating elementary units of quantum information in the corresponding basis states of a single unit of quantum information and thus relating different states within a tensor space of many units of quantum information. The basis states within this tensor space are given by the number of units of quantum information, which are in the four basis states of a single unit of quantum information, $\left|N_{a}, N_{b}, N_{c}, N_{d}\right\rangle$. Accordingly these states are related to each other as follows:

$$
\begin{aligned}
\hat{a}\left|N_{a}, N_{b}, N_{c}, N_{d}\right\rangle & =\sqrt{N_{a}}\left|N_{a}-1, N_{b}, N_{c}, N_{d}\right\rangle, \\
\hat{a}^{\dagger}\left|N_{a}, N_{b}, N_{c}, N_{d}\right\rangle & =\sqrt{N_{a}+1}\left|N_{a}+1, N_{b}, N_{c}, N_{d}\right\rangle, \\
\hat{b}\left|N_{a}, N_{b}, N_{c}, N_{d}\right\rangle & =\sqrt{N_{b}}\left|N_{a}, N_{b}-1, N_{c}, N_{d}\right\rangle, \\
\hat{b}^{\dagger}\left|N_{a}, N_{b}, N_{c}, N_{d}\right\rangle & =\sqrt{N_{b}+1}\left|N_{a}, N_{b}+1, N_{c}, N_{d}\right\rangle, \\
\hat{c}\left|N_{a}, N_{b}, N_{c}, N_{d}\right\rangle & =\sqrt{N_{c}}\left|N_{a}, N_{b}, N_{c}-1, N_{d}\right\rangle, \\
\hat{c}^{\dagger}\left|N_{a}, N_{b}, N_{c}, N_{d}\right\rangle & =\sqrt{N_{c}+1}\left|N_{a}, N_{b}, N_{c}+1, N_{d}\right\rangle, \\
\hat{d}\left|N_{a}, N_{b}, N_{c}, N_{d}\right\rangle & =\sqrt{N_{d}}\left|N_{a}, N_{b}, N_{c}, N_{d}-1\right\rangle, \\
\hat{d}^{\dagger}\left|N_{a}, N_{b}, N_{c}, N_{d}\right\rangle & =\sqrt{N_{d}+1}\left|N_{a}, N_{b}, N_{c}, N_{d}+1\right\rangle .
\end{aligned}
$$

They are created from a vacuum state $|0\rangle$ containing no quantum information and being defined by the condition

$$
\hat{a}|0\rangle=\hat{b}|0\rangle=\hat{c}|0\rangle=\hat{d}|0\rangle=0
$$


One obtains an arbitrary basis state in the tensor space, $\left|N_{a}, N_{b}, N_{c}, N_{d}\right\rangle$, if the corresponding creation operators $\hat{a}^{\dagger}, \hat{b}^{\dagger}, \hat{c}^{\dagger}$ and $\hat{d}^{\dagger}$ are applied $N_{a}, N_{b}, N_{c}$ and $N_{d}$ times to the vacuum state $|0\rangle$,

$$
\left|N_{a}, N_{b}, N_{c}, N_{d}\right\rangle=\frac{\hat{a}^{\dagger N_{a}} \hat{b}^{\dagger N_{b}} \hat{c}^{\dagger N_{c}} \hat{d}^{\dagger N_{d}}}{\sqrt{N_{a} !} \sqrt{N_{b} !} \sqrt{N_{c} !} \sqrt{N_{d} !}}|0\rangle
$$

The commutation relations of the creation and annihilation operators (3) imply that the units of quantum information obey Bose statistics. If they would obey Fermi statistics, there could according to the Pauli exclusion principle only exist states containing not more than four units of quantum information corresponding to the four possible basis states of one single unit. A basis state in the tensor space can be represented as the tensor product of four independent states, $\left|N_{a}\right\rangle,\left|N_{b}\right\rangle,\left|N_{c}\right\rangle$ and $\left|N_{d}\right\rangle$, referring to each pair of creation and annihilation operators. These states are eigenstates of the four occupation number operators referring to the four basis states of a single unit of quantum information and thus fulfil the following eigenvalue equations:

$$
\begin{aligned}
& \hat{a}^{\dagger} \hat{a}\left|N_{a}\right\rangle=N_{a}\left|N_{a}\right\rangle \quad, \quad \hat{b}^{\dagger} \hat{b}\left|N_{b}\right\rangle=N_{b}\left|N_{b}\right\rangle, \\
& \hat{c}^{\dagger} \hat{c}\left|N_{c}\right\rangle=N_{c}\left|N_{c}\right\rangle \quad, \quad \hat{d}^{\dagger} \hat{d}\left|N_{d}\right\rangle=N_{d}\left|N_{d}\right\rangle \text {. }
\end{aligned}
$$

The inner product of these eigenstates with each other is defined according to

$$
\begin{aligned}
& \left\langle N_{a}^{\prime} \mid N_{a}\right\rangle=\delta_{N_{a} N_{a}^{\prime}} \quad, \quad\left\langle N_{b}^{\prime} \mid N_{b}\right\rangle=\delta_{N_{b} N_{b}^{\prime}} \\
& \left\langle N_{c}^{\prime} \mid N_{c}\right\rangle=\delta_{N_{c} N_{c}^{\prime}} \quad, \quad\left\langle N_{d}^{\prime} \mid N_{d}\right\rangle=\delta_{N_{d} N_{d}^{\prime}}
\end{aligned}
$$

Since a basis state in the complete tensor space can be expressed as the tensor product of the eigenstates of the four occupation number operators defined in (7),

$$
\left|N_{a}, N_{b}, N_{c}, N_{d}\right\rangle=\left|N_{a}\right\rangle \otimes\left|N_{b}\right\rangle \otimes\left|N_{c}\right\rangle \otimes\left|N_{d}\right\rangle,
$$

the inner product of two basis states in the complete tensor space can accordingly be expressed as

$$
\begin{aligned}
& \left\langle N_{a}^{\prime}, N_{b}^{\prime}, N_{c}^{\prime}, N_{d}^{\prime} \mid N_{a}, N_{b}, N_{c}, N_{d}\right\rangle \\
= & \left\langle N_{a}^{\prime} \mid N_{a}\right\rangle\left\langle N_{b}^{\prime} \mid N_{b}\right\rangle\left\langle N_{c}^{\prime} \mid N_{c}\right\rangle\left\langle N_{d}^{\prime} \mid N_{d}\right\rangle \\
= & \delta_{N_{a}^{\prime} N_{a}} \delta_{N_{b}^{\prime} N_{b}} \delta_{N_{c}^{\prime} N_{c}} \delta_{N_{d}^{\prime} N_{d}} .
\end{aligned}
$$

A general state $|\Psi\rangle$ in the Hilbert space of many units of quantum information corresponds of course to an arbitrary superposition of the basis states,

$$
|\Psi\rangle=\sum_{N_{a}, N_{b}, N_{c}, N_{d}} c\left(N_{a}, N_{b}, N_{c}, N_{d}\right)\left|N_{a}, N_{b}, N_{c}, N_{d}\right\rangle
$$

where $c\left(N_{a}, N_{b}, N_{c}, N_{d}\right)$ denotes arbitrary coefficients, which can be described as projections on the general state to the basis states,

$$
c\left(N_{a}, N_{b}, N_{c}, N_{d}\right)=\left\langle N_{a}, N_{b}, N_{c}, N_{d} \mid \Psi\right\rangle .
$$

To be able to give a more clear arranged notation, it will be used the following notation for a general state below:

$$
|\Psi\rangle=\sum_{N} c(N)|N\rangle \quad, \quad N \widehat{=}\left(N_{a}, N_{b}, N_{c}, N_{d}\right) .
$$

By using (10), (11) and (13) the inner product of two general states in the tensor space can be written as

$$
\begin{aligned}
\langle\chi \mid \psi\rangle & =\sum_{N^{\prime}} \sum_{N} d^{*}\left(N^{\prime}\right) c(N)\left\langle N^{\prime} \mid N\right\rangle \\
& =\sum_{N^{\prime}} \sum_{N} d^{*}\left(N^{\prime}\right) c(N) \delta_{N^{\prime} N} \\
& =\sum_{N} d^{*}(N) c(N) .
\end{aligned}
$$

\section{CONSTRUCTION OF POSITION AND MOMENTUM OPERATORS OF SINGLE PARTICLES}

By introducing the new components $a_{x}, a_{y}, a_{z}$ and $a_{t}$ being defined by the components $a, b, c$ and $d$ as follows:

$$
\begin{aligned}
a_{x} & =\frac{1}{2}(a-b+c-d), & a_{y} & =\frac{1}{2}(a-b-c+d), \\
a_{z} & =\frac{1}{2}(a+b-c-d), & a_{t} & =\frac{1}{2}(a+b+c+d),
\end{aligned}
$$

the Weyl spinor $u$ describing an elementary unit of quantum information can be expressed as

$u=\left(\begin{array}{l}\frac{1}{2}\left(a_{x}+a_{y}+a_{z}+a_{t}\right)+\frac{1}{2}\left(-a_{x}-a_{y}+a_{z}+a_{t}\right) i \\ \frac{1}{2}\left(a_{x}-a_{y}-a_{z}+a_{t}\right)+\frac{1}{2}\left(-a_{x}+a_{y}-a_{z}+a_{t}\right) i\end{array}\right)$.

The new components $a_{x}, a_{y}, a_{z}$ and $a_{t}$ refer to linear combinations of eigenvectors to the Pauli matrices

$\sigma_{x}=\left(\begin{array}{ll}0 & 1 \\ 1 & 0\end{array}\right), \sigma_{y}=\left(\begin{array}{cc}0 & -i \\ i & 0\end{array}\right), \sigma_{z}=\left(\begin{array}{cc}1 & 0 \\ 0 & -1\end{array}\right), \sigma_{t}=\left(\begin{array}{ll}1 & 0 \\ 0 & 1\end{array}\right)$,

in the Weyl spinor space of an elementary unit of quantum information. After quantization the corresponding operators $\hat{a}_{x}, \hat{a}_{y}, \hat{a}_{z}$ and $\hat{a}_{t}$ fulfil the same commutation relations as the operators $\hat{a}, \hat{b}, \hat{c}$ and $\hat{d}$,

$\left[\hat{a}_{x}, \hat{a}_{x}^{\dagger}\right]=1, \quad\left[\hat{a}_{y}, \hat{a}_{y}^{\dagger}\right]=1, \quad\left[\hat{a}_{z}, \hat{a}_{z}^{\dagger}\right]=1, \quad\left[\hat{a}_{t}, \hat{a}_{t}^{\dagger}\right]=1$, 
and therefore constitute the same tensor space referring to elementary quantum information, which has been considered in the last section, but now with respect to a new basis in the Weyl spinor space of a single elementary unit of quantum information. The corresponding new basis states of the tensor space are accordingly defined by the occupation numbers $N_{x}, N_{y}, N_{z}$ and $N_{t}$. The properties of the tensor space remain exactly the same as considered in the last section. From the annihilation operators $\hat{a}_{x}$, $\hat{a}_{y}, \hat{a}_{z}$ and $\hat{a}_{t}$ and the corresponding creation operators $\hat{a}_{x}^{\dagger}, \hat{a}_{y}^{\dagger}, \hat{a}_{z}^{\dagger}$ and $\hat{a}_{t}^{\dagger}$ can now be defined hermitian operators according to

$$
\begin{array}{lll}
\hat{x}=\frac{1}{\sqrt{2}}\left(\hat{a}_{x}+\hat{a}_{x}^{\dagger}\right) \quad, & \hat{p}_{x}=-\frac{i}{\sqrt{2}}\left(\hat{a}_{x}-\hat{a}_{x}^{\dagger}\right), \\
\hat{y}=\frac{1}{\sqrt{2}}\left(\hat{a}_{y}+\hat{a}_{y}^{\dagger}\right), & \hat{p}_{y}=-\frac{i}{\sqrt{2}}\left(\hat{a}_{y}-\hat{a}_{y}^{\dagger}\right), \\
\hat{z}=\frac{1}{\sqrt{2}}\left(\hat{a}_{z}+\hat{a}_{z}^{\dagger}\right), & \hat{p}_{z}=-\frac{i}{\sqrt{2}}\left(\hat{a}_{z}-\hat{a}_{z}^{\dagger}\right), \\
\hat{t}=\frac{1}{\sqrt{2}}\left(\hat{a}_{t}+\hat{a}_{t}^{\dagger}\right), & \hat{p}_{t}=-\frac{i}{\sqrt{2}}\left(\hat{a}_{t}-\hat{a}_{t}^{\dagger}\right),
\end{array}
$$

fulfilling a Heisenberg algebra like position and momentum operators,

$$
\left[\hat{x}, \hat{p}_{x}\right]=i, \quad\left[\hat{y}, \hat{p}_{y}\right]=i, \quad\left[\hat{z}, \hat{p}_{z}\right]=i, \quad\left[\hat{t}, \hat{p}_{t}\right]=i,
$$

which are therefore isomorphic to such operators. It is now a postulate to identify these operators with the real position and momentum operators referring to Minkowski space-time. If one wants conversely to express the creation and annihilation operators by the position and momentum operators, one obtains the following equations:

$$
\begin{aligned}
& \hat{a}_{x}=\frac{1}{\sqrt{2}}\left(\hat{x}+i \hat{p}_{x}\right) \quad, \quad \hat{a}_{x}^{\dagger}=\frac{1}{\sqrt{2}}\left(\hat{x}-i \hat{p}_{x}\right), \\
& \hat{a}_{y}=\frac{1}{\sqrt{2}}\left(\hat{y}+i \hat{p}_{y}\right) \quad, \quad \hat{a}_{y}^{\dagger}=\frac{1}{\sqrt{2}}\left(\hat{y}-i \hat{p}_{y}\right), \\
& \hat{a}_{z}=\frac{1}{\sqrt{2}}\left(\hat{z}+i \hat{p}_{z}\right) \quad, \quad \hat{a}_{z}^{\dagger}=\frac{1}{\sqrt{2}}\left(\hat{z}-i \hat{p}_{z}\right), \\
& \hat{a}_{t}=\frac{1}{\sqrt{2}}\left(\hat{t}+i \hat{p}_{t}\right) \quad, \quad \hat{a}_{t}^{\dagger}=\frac{1}{\sqrt{2}}\left(\hat{t}-i \hat{p}_{t}\right) .
\end{aligned}
$$

Of course the position and momentum operators constructed by the creation and annihilation operators referring to the abstract tensor space of elementary quantum information (19) lead to Minkowski space-time, since they can naturally be represented in Minkowski spacetime. By referring to this representation in Minkowski space-time, the creation and annihilation operators look as follows:

$$
\begin{array}{cc}
\hat{a}_{x}=\frac{1}{\sqrt{2}}\left(x+\frac{\partial}{\partial x}\right), & \hat{a}_{x}^{\dagger}=\frac{1}{\sqrt{2}}\left(x-\frac{\partial}{\partial x}\right), \\
\hat{a}_{y}=\frac{1}{\sqrt{2}}\left(y+\frac{\partial}{\partial y}\right), & \hat{a}_{y}^{\dagger}=\frac{1}{\sqrt{2}}\left(y-\frac{\partial}{\partial y}\right), \\
\hat{a}_{z}=\frac{1}{\sqrt{2}}\left(z+\frac{\partial}{\partial z}\right), & \hat{a}_{z}^{\dagger}=\frac{1}{\sqrt{2}}\left(z-\frac{\partial}{\partial z}\right), \\
\hat{a}_{t}=\frac{1}{\sqrt{2}}\left(t+\frac{\partial}{\partial t}\right), & \hat{a}_{t}^{\dagger}=\frac{1}{\sqrt{2}}\left(t-\frac{\partial}{\partial t}\right) .
\end{array}
$$

\section{TRANSITION FROM STATES IN TENSOR SPACE TO STATES IN SPACE-TIME}

In the last section it has been shown that it is possible to map the four pairs of creation and annihilation operators acting in the tensor space referring to a Weyl spinor as basis space to position and momentum operators referring to a Minkowski space-time. This means that the tensor space of elementary quantum information is isomorphic to Minkowski space-time and therefore it has to be possible to represent the states in the tensor space as wave functions on Minkowski space-time. To obtain the representation in Minkowski space-time there has to be used the mathematical description of the more dimensional quantum theoretical harmonic oscillator. The harmonic oscillator can be described by introduction of creation and annihilation operators, which are related to the position and momentum operators according to the consideration of this paper (19), (21). The Hamilton operator of the harmonic oscillator corresponds then to the occupation number operator and an additional vacuum expectation value and the eigenstates of the Hamilton operator can be represented in position space by using the hermite polynoms. In the scenario of this paper is defined no Hamilton operator, but the eigenstates of the Hamilton operator of the harmonic oscillator correspond to the basis states in the tensor space, which can therefore be represented in position space, in Minkowski space-time in this case to be more specific. One has to begin with the separated eigenstates of the occupation number operators, which can be represented as in case of the harmonic oscillator in position space according to

$$
\begin{aligned}
& \varphi_{N_{x}}(x)=\left\langle x \mid N_{x}\right\rangle=\frac{1}{\left(N_{x} ! 2^{N_{x}} \pi^{2}\right)}\left(x-\frac{\partial}{\partial x}\right)^{N_{x}} \exp \left(-\frac{x^{2}}{2}\right), \\
& \varphi_{N_{y}}(y)=\left\langle y \mid N_{y}\right\rangle=\frac{1}{\left(N_{y} ! 2^{N_{y}} \pi^{2}\right)}\left(y-\frac{\partial}{\partial y}\right)^{N_{y}} \exp \left(-\frac{y^{2}}{2}\right), \\
& \varphi_{N_{z}}(z)=\left\langle z \mid N_{z}\right\rangle=\frac{1}{\left(N_{z} ! 2^{N_{z}} \pi^{2}\right)}\left(z-\frac{\partial}{\partial z}\right)^{N_{z}} \exp \left(-\frac{z^{2}}{2}\right), \\
& \varphi_{N_{t}}(t)=\left\langle t \mid N_{t}\right\rangle=\frac{1}{\left(N_{t} ! 2^{N_{t}} \pi^{2}\right)}\left(t-\frac{\partial}{\partial t}\right)^{N_{t}} \exp \left(-\frac{t^{2}}{2}\right)(23)
\end{aligned}
$$

An arbitrary state in the complete tensor space can then accordingly be represented as a superposition of the ten- 
sor product of these projections to the eigenstates of the four position operators

$$
\begin{aligned}
& \Psi(x, y, z, t)=\langle x, y, z, t \mid \Psi\rangle \\
= & \sum_{N} c\left(N_{x}, N_{y}, N_{z}, N_{t}\right)\left\langle x \mid N_{x}\right\rangle\left\langle y \mid N_{y}\right\rangle\left\langle z \mid N_{z}\right\rangle\left\langle t \mid N_{t}\right\rangle \\
= & \sum_{N} c\left(N_{x}, N_{y}, N_{z}, N_{t}\right) \varphi_{N_{x}}(x) \varphi_{N_{y}}(y) \varphi_{N_{z}}(z) \varphi_{N_{t}}(t),
\end{aligned}
$$

where it has been defined: $|x, y, z, t\rangle=|x\rangle \otimes|y\rangle \otimes|z\rangle \otimes|t\rangle$. This means that a general state in the tensor space (11) is isomorphic to a wave function in Minkowski space-time. Below a general state in the tensor space represented as a wave function in Minkowski space-time will be written as following:

$$
\Psi(X)=\Psi(x, y, z, t) \quad, \quad X=(x, y, z, t),
$$

and the product of the eigenstates of the four occupation number operators will be expressed as

$$
\varphi_{N}(X)=\varphi_{N_{x}}(x) \varphi_{N_{y}}(y) \varphi_{N_{z}}(z) \varphi_{N_{t}}(t)
$$

\section{REPRESENTATION OF THE POINCARE GROUP IN TENSOR SPACE}

If a general state in the tensor space can be represented in Minkowski space-time, it has to be possible to represent the Poincare group in the tensor space. In this section there shall be given the representation of the Poincare group in the tensor space. A transformation in the tensor space yields then a corresponding transformation concerning the representation of the state in Minkowski space-time. The Poincare group consists of the four space-time translations, the rotations in the three-dimensional spatial subspace of Minkowski spacetime and the Lorentz boosts referring to the the three spatial directions. Thus it is described by ten generators. The four generators of space-time translations are the momentum operators and the energy operator given in (19),

$$
\begin{aligned}
& P_{0}=\hat{p}_{t}=-\frac{i}{\sqrt{2}}\left(\hat{a}_{t}-\hat{a}_{t}^{\dagger}\right), \\
& P_{1}=\hat{p}_{x}=-\frac{i}{\sqrt{2}}\left(\hat{a}_{x}-\hat{a}_{x}^{\dagger}\right), \\
& P_{2}=\hat{p}_{y}=-\frac{i}{\sqrt{2}}\left(\hat{a}_{y}-\hat{a}_{y}^{\dagger}\right), \\
& P_{3}=\hat{p}_{z}=-\frac{i}{\sqrt{2}}\left(\hat{a}_{z}-\hat{a}_{z}^{\dagger}\right) .
\end{aligned}
$$

From these operators there can be constructed the generators of the Lorentz group consisting of the three angular momentum operators generating the three-dimensional rotation group and the three Lorentz boost generators,

$$
\begin{aligned}
& M_{23}=\hat{y} \hat{p}_{z}-\hat{z} \hat{p}_{y}=i\left(\hat{a}_{y}^{\dagger} \hat{a}_{z}-\hat{a}_{z}^{\dagger} \hat{a}_{y}\right), \\
& M_{13}=\hat{z} \hat{p}_{x}-\hat{x} \hat{p}_{z}=i\left(\hat{a}_{z}^{\dagger} \hat{a}_{x}-\hat{a}_{x}^{\dagger} \hat{a}_{z}\right), \\
& M_{12}=\hat{x} \hat{p}_{y}-\hat{y} \hat{p}_{x}=i\left(\hat{a}_{x}^{\dagger} \hat{a}_{y}-\hat{a}_{y}^{\dagger} \hat{a}_{x}\right) \\
& M_{01}=\hat{t} \hat{p}_{x}+\hat{x} \hat{p}_{t}=i\left(\hat{a}_{t} \hat{a}_{x}-\hat{a}_{x}^{\dagger} \hat{a}_{t}^{\dagger}\right) \\
& M_{02}=\hat{t} \hat{p}_{y}+\hat{y} \hat{p}_{t}=i\left(\hat{a}_{t} \hat{a}_{y}-\hat{a}_{y}^{\dagger} \hat{a}_{t}^{\dagger}\right) \\
& M_{03}=\hat{t} \hat{p}_{z}+\hat{z} \hat{p}_{t}=i\left(\hat{a}_{t} \hat{a}_{z}-\hat{a}_{z}^{\dagger} \hat{a}_{t}^{\dagger}\right)
\end{aligned}
$$

The generators of the Poincare group fulfil the following Lie Algebra defining its characteristic group structure,

$$
\begin{array}{rlr}
{\left[P_{\mu}, P_{\nu}\right]} & =0, & \mu, \nu, \rho, \sigma=0 \ldots 3, \\
{\left[M_{\mu \nu}, P_{\rho}\right]} & =i \eta_{\mu \rho} P_{\nu}-i \eta_{\nu \rho} P_{\mu}, \\
{\left[M_{\mu \nu}, M_{\rho \sigma}\right]} & =-i \eta_{\mu \rho} M_{\nu \sigma}+i \eta_{\mu \sigma} M_{\nu \rho}-i \eta_{\nu \rho} M_{\mu \sigma}+i \eta_{\nu \sigma} M_{\mu \rho} .
\end{array}
$$

This means that a general Poincare transformation applied to a general quantum state in the tensor space is of the following shape:

$$
\begin{aligned}
|\Psi\rangle \rightarrow \quad & \exp \left[i a_{\mu} P^{\mu}\left(\hat{a}, \hat{a}^{\dagger}\right)+i \omega_{\mu \nu} M^{\mu \nu}\left(\hat{a}, \hat{a}^{\dagger}\right)\right]|\Psi\rangle \\
& =\sum_{N} c(N) \exp \left[i a_{\mu} P^{\mu}\left(\hat{a}, \hat{a}^{\dagger}\right)+i \omega_{\mu \nu} M^{\mu \nu}\left(\hat{a}, \hat{a}^{\dagger}\right)\right]|N\rangle
\end{aligned}
$$

\section{QUANTUM FIELD THEORY}

In the last section an arbitrary state in the tensor space of elementary quantum information has been mapped to four-dimensional space-time where can be represented the Lorentz group and thus it has been mapped to Minkowski space-time. The resulting state can be considered as a wave function describing a particle. A basis state in the tensor space is defined by the occupation number with respect to the four basis states of a single unit of quantum information. To obtain a description of quantum field theory, which is based on the elementary quantum information, the wave function as representation of a general state in the tensor space has to become an operator by itself, which acts on states in a new Hilbert space. The wave function obtained as representation of a state in the Hilbert space of many units of elementary quantum information in the consideration developed here is transformed to an operator by postulating commutation relations of the coefficients defining a general state in tensor space as superposition of the corresponding basis states, 


$$
\left[\hat{c}(N), \hat{c}^{\dagger}\left(N^{\prime}\right)\right]=i \delta_{N N^{\prime}}
$$

Thus the coefficients become operators $\hat{c}(N)$, which behave as annihilation operators in a new Hilbert space. The corresponding hermitian adjoint operators $\hat{c}^{\dagger}(N)$ behave accordingly as creation operators. The wave function in space-time acts after quantization as an operator in this Hilbert space,

$$
\hat{\Psi}(x, y, z, t)=\sum_{N} \hat{c}(N) \varphi_{N_{x}}(x) \varphi_{N_{y}}(y) \varphi_{N_{z}}(z) \varphi_{N_{t}}(t) .
$$

By using (25) it can be represented as

$$
\hat{\Psi}(X)=\sum_{N} \hat{c}(N) \varphi_{N}(X) .
$$

The corresponding vacuum state, $|0\rangle_{\mathcal{N}}$, is defined by

$$
\hat{c}(N)|0\rangle_{\mathcal{N}}=0, \forall N .
$$

The basis states of the Hilbert space of quantum field theory, $|\mathcal{N}\rangle$, defined by the number of particles in the corresponding basis states of the tensor space, which is according to the considerations above defined by a distribution of quantum information in the several states of a single unit of quantum information, can be obtained by applying the corresponding creation operators for particles, $\hat{c}(N)^{\dagger}, \mathcal{N}(N)$ times to the vacuum state $|0\rangle_{\mathcal{N}}$,

$$
|\mathcal{N}\rangle=\sum_{N} \hat{c}^{\dagger}(N)^{\mathcal{N}(N)}|0\rangle_{\mathcal{N}}
$$

The corresponding scalar product between these basis states is accordingly defined as

$$
\left\langle\mathcal{N}^{\prime} \mid \mathcal{N}\right\rangle=\prod_{N} \delta_{\mathcal{N}^{\prime}(N) \mathcal{N}(N)}=\delta_{\mathcal{N}^{\prime} \mathcal{N}}
$$

A general state in the Hilbert space of many particle theory or quantum field theory is defined by a general superposition of basis states (35) in the following way:

$$
|\Phi\rangle=\sum_{\mathcal{N}} \mathcal{C}(\mathcal{N})|\mathcal{N}\rangle
$$

It is also possible to formulate a propagator within the tensor space, $\Delta\left(X, X^{\prime}\right)$, which is defined as

$$
\Delta\left(X, X^{\prime}\right)=\left\langle 0\left|\hat{\Psi}(X) \hat{\Psi}^{\dagger}\left(X^{\prime}\right)\right| 0\right\rangle .
$$

This expression for the propagator can be transformed by the following calculation:

$$
\begin{aligned}
\Delta\left(X, X^{\prime}\right) & =\left\langle 0\left|\hat{\Psi}(X) \hat{\Psi}^{\dagger}\left(X^{\prime}\right)\right| 0\right\rangle \\
& =\sum_{N, N^{\prime}} \varphi_{N}(X) \varphi_{N^{\prime}}^{*}\left(X^{\prime}\right)\left\langle 0\left|\hat{c}(N) \hat{c}^{\dagger}\left(N^{\prime}\right)\right| 0\right\rangle \\
& =\sum_{N, N^{\prime}} \varphi_{N}(X) \varphi_{N^{\prime}}^{*}\left(X^{\prime}\right)\left\langle\Phi_{\mathcal{N}(N)=1} \mid \Phi_{\mathcal{N}\left(N^{\prime}\right)=1}^{\prime}\right\rangle \\
& =\sum_{N, N^{\prime}} \varphi_{N}(X) \varphi_{N^{\prime}}^{*}\left(X^{\prime}\right) \delta_{N, N^{\prime}} \\
& =\sum_{N} \varphi_{N}(X) \varphi_{N}^{*}\left(X^{\prime}\right),
\end{aligned}
$$

where $\varphi_{N}(X)$ describes according to (26) the representation of a basis state in the tensor space as wave function in space-time. In the calculation (39) has been used the following notation for a state of a single particle expressed in the Hilbert space of quantum field theory:

$$
\left|\Phi_{\mathcal{N}(M)=1}\right\rangle \widehat{=}\left\{\begin{array}{l}
\mathcal{N}(N)=1, \text { if } \mathrm{N}=\mathrm{M} \\
\mathcal{N}(N)=0 \text { else }
\end{array}\right.
$$

\section{CONCEPTUAL ISSUES}

Both theories of contemporary fundamental physics, quantum theory as well as general relativity, suggest that physical reality is nonlocal on a fundamental level. In general relativity the mathematical property of diffeomorphism invariance is connected to the relationalistic attitude with respect to space-time, which is suggested by the theory. In quantum theory the mathematical formalism and many phenomena unambiguously reflect such a nonlocal property of nature. The postulates of general quantum theory within the mathematical formulation given by Paul Adrien Maurice Dirac and Johann von Neumann [13], 14] do not presuppose position space. This is the reason why it was possible to deal with a quantum theory of abstract quantum information without presupposing space-time in this paper, but to represent the obtained states in a mathematical space being isomorphic to real space-time and to postulate that this representation space corresponds to real space-time. Phenomena like the Einstein Podolsky Rosen paradoxon or the double slit experiment show with respect to concrete experiments that physical reality as it is described by quantum theory is nonlocal. In a state, where two particles moving into two opponent directions are correlated with each other, the measurement of an observable of one particle instantaneously influences the complete state and thus also the other particle. In the double slit experiment with single particles the question through which slit the particle has moved already leads to contradictions with the experiment and thus the concept of a trajectory of a particle is not appropriate concerning quantum theoretical phenomena. If these phenomena would be interpreted as phenomena, which presuppose 
the local causal structure of space-time, they would explicitly contradict special relativity, since they contain an instantaneous exchange of physical information. Therefore it seems to be necessary to assume that they refer to a level of reality where this structure must not to be presupposed yet, but that this more fundamental quantum theoretical reality constitutes the macroscopic local causal structure of space-time, which thus has to be derived from quantum theory. Since the mathematical formalism of general quantum theory seems to describe physics in the microcosm as far as it is known correctly, it is not astonishing that the nonlocal character of quantum theory is contained in the mathematical formalism as well as the concrete phenomena. The one is just the general theoretical representation of the other. If spacetime is not considered as fundamental, then particles and fields as they are presupposed in particle physics have to be replaced by a more fundamental entity. This entity could be the elementary quantum information, which is constitutive within Carl Friedrich von Weizsaeckers idea of a reconstruction of physics containing the concept of alternatives as general shape of human knowledge about nature. The description of physical states by using elementary quantum information does not presuppose space-time, but leads to a description of physical reality implicitly containing the structure of real spacetime. Accordingly space-time is considered as a kind of representation of a more fundamental reality of quantum theoretical relations, which can be expressed by quantum information resolved into elementary units of quantum information. The conceptual issues concerning this question of the nature of space-time are more elaborately treated in [1] [2], [3], [4], 15], [16].

\section{SUMMARY AND DISCUSSION}

In this paper has been considered quantum field theory within a representation by elementary quantum information. There has been defined a tensor space of quantum information as it appears within the quantum theory of ur-alternatives of Carl Friedrich von Weizsaecker, which postulates abstract quantum information as fundamental entity of nature. From the components of the quantized units of quantum information described by Weyl spinors, which become operators annihilating units of quantum information in the corresponding basis state and thus constituting the tensor space, have been defined position and momentum operators. It has been shown that by referring to these operators and using the eigenstates of the harmonic oscillator represented in position space it is possible to perform a transition from a general state in the tensor space of quantum information to position space. This means that a general state in the tensor space can be represented as a wave function in Minkowski space-time describing the state of a single particle. Another quantization of the amplitudes of the states in the tensor space leads to many particle theory corresponding to quantum field theory. The theory gives the possibility to obtain a description of quantum field theory related to a more fundamental purely quantum theoretical and thus nonlocal reality, which constitutes physical objects appearing in space-time. Since the tensor space of elementary quantum information is discrete, it could become possible to avoid the appearance of divergences from the beginning.
[1] C. F. von Weizsaecker, "Komplementaritt und Logik," Die Naturwissenschaften 42, 521-529, 545-555 (1955). C. F. von Weizsaecker, "Komplementaritt und Logik, II. Die Quantentheorie der einfachen Alternative," Zeitschrift fr Naturforschung 13a, 245-253 (1958).

C. F. von Weizsaecker, E. Scheibe und G. Suessmann, "Komplementaritt und Logik, III. Mehrfache Quantelung," Zeitschrift fr Naturforschung 13a, 705 (1958).

[2] C. F. von Weizsaecker, Die Einheit der Natur, Carl Hanser Verlag, Muenchen, 1971.

[3] C. F. von Weizsaecker, Aufbau der Physik, Carl Hanser Verlag, Muenchen, 1985.

[4] C. F. von Weizsaecker, Zeit und Wissen, Carl Hanser Verlag, Muenchen, 1992.

[5] T. Goernitz, D. Graudenz und C. F. von Weizsaecker, "Quantum Field Theory of Binary Alternatives," Int. J. Theor. Phys. 31, 1929-1959 (1992).

[6] H. Lyre, Int. J. Theor. Phys. 34 (1995) 1541 arXiv:quant-ph/9611048.

[7] H. Lyre, Int. J. Theor. Phys. 35 (1996) 2219 arXiv:quant-ph/9702047.

[8] H. Lyre, In ${ }^{*}$ Castell, L. (ed.) et al.: Time, quantum and information* 373-383.
[9] R. Penrose and W. Rindler, Spinors and space-time. Vol. 1: Two spinor calculus and relativistic fields, Cambridge University Press, Cambridge 1984, p. 458.

[10] R. Penrose and W. Rindler, Spinors and space-time. Vol. 2: Spinor and twistor methods in space-time, Cambridge University Press, Cambridge 1986, p. 501.

[11] E. P. Wigner, Annals of Mathematics 40 (1939), 149-204

[12] S. Weinberg, The Quantum theory of fields. Vol. 1: Foundations, Cambridge University Press, Cambridge 1995, p. 609.

[13] P.A.M. Dirac, The principles of quantum mechanics, Oxford University Press, Oxford 1958.

[14] J. von Neumann, Mathematische Grundlagen der Quantenmechanik, Berlin 1932.

[15] M. Kober, "Copenhagen Interpretation of Quantum Theory and the Measurement Problem," arXiv:0905.0408.

[16] M. Kober, Die Konstituierung der Raum-Zeit in einer einheitlichen Naturtheorie. Ueber die Beziehung der begrifflichen Grundlagen der Quantentheorie und der Allgemeinen Relativitaetstheorie, Suedwestdeutscher Verlag fuer Hochschulschriften, Saarbruecken 2011. 